\title{
CORONAL LOOP INTERACTION
}

\author{
Raymond N. Smartt and Zhenda Zhang 1 \\ National Solar Observatory/Sacramento Peak \\ Sunspot, New Mexico 88349 U.S.A.
}

\begin{abstract}
High-resolution images of post-flare loop systems in Fe XIV (5303 $)$ and $\mathrm{FeX}(6374 \AA)$ display transient enhancements at the projected intersections of some loops. The brightness of such enhancements gradually increases to a marked maximum and then fades with a typical lifetime of about thirty minutes. The maximum in the red line lags that of the green line by about ten minutes, while $\mathrm{Ha}$ reaches a maximum about ten minutes later, which gives a measure of the cooling time, and hence electron density. The observed phenomenon is interpreted as localized loop coalescence and partial magnetic reconnection, with the possibility of an increase in the current density, and heating of the plasma in the immediate vicinity of the $\mathrm{X}$-point.
\end{abstract}

The coalescence of current loops leading to magnetic reconnection has been widely studied in relation to flares (see, for example, Tajima et al, 1987 and Tajima et al, 1982). In such treatments, internally-and externally-driven magnetic reconnection of the plasma is considered over different time scales and energies. Here we discuss the case of externally-driven reconnection in the low-energy case of post-flare coronal loops.

The data are from the 20-cm aperture NSO/SP emission-line coronagraph that records photographically sequential images in Fe XIV (5303 $\AA$ ), FeX $(6374 \AA)$ and $\mathrm{H} a$, with a normal cadence of one minute. High-resolution post-flare loop images in the green line show occasional marked enhancements in the vicinity of the projected intersection of two loops. From the data it is evident that such enhancements occur as one loop develops or moves to intersect a neighbor, producing an X-point of coalescence. The morphology of such events as seen at their maximum, especially clear in cases where the plane of the loops are not strongly inclined to the line-of-sight, is a brightness at the point of intersection far greater than the simple sum of the brightness of the individual loops, and some enhancement extending partially along the loops away from the intersection point. Further, there is some partial "filling in" of the space between the loops, marked by a sharp boundary, evidently a process of redistribution of magnetic flux between non-aligned loops, with partial magnetic reconnection. Observations of over 80 events have been observed. It is found that typical enhancements increase to a

1On leave from Department of Astronomy, University of Nanjing, Nanjing, PRC.

E. R. Priest and V. Krishan (eds.), Basic Plasma Processes on the Sun, 350-351.

(C) 1990 IAU. Printed in the Netherlands. 
maximum over a period on average of about 15 minutes and then gradually fade with a lifetime of the order of 30 minutes. Usually at least several such events are observed in a post-flare loop development. The maximum brightness varies between different events, but all those identified as such show a brightness obviously significantly greater than the loops in gemera;. This leaves the possibility that there is a regime of weaker events that remain unidentified in data of this quality. Further, events with small spatial scale would probably not be detected except in exceptionally high-resolution data. It appears therefore that this is a relatively common phenomenon as detected in the visible emission corona, especially around solar maximum with an average of at least several events per day. Corresponding enhancements at the same location appear in the FeX (6374 $\AA$ ) images. The overall red-line emission image is generally much fainter than that of the green line, and the enhancements in the red-line are correspondingly weaker, but nevertheless are usually well-defined in the form of a highly localized bright patch. Each maximum lags the corresponding maximum in the green line by a few minutes, with a mean of about 10 minutes, a measure of the cooling time. About 10 minutes later again, $\mathrm{H} \alpha$ reaches a maximum at the site of the reconnection. In some cases it is completely absent just minutes before. It should be pointed out that a post-flare loop event is itself a highly dynamical system in which the loops, typically in a complex configuration, gradually increase in height. During this time period of several hours, new loops can appear, while others disappear, with a systematic trend to simpler configurations, a decreasing number of loops and fainter emission. Apparently it is this dynamical property of post-flare loops that strongly increases the probability of loop coalescence, as compared with coronal loops in general. But the dynamical property of post-flare loops also results in some uncertainty in tracing the morphology of a loop coalescence event through its life. Nevertheless, the characteristics of the enhancements as described above represent a very well-defined phenomenon.

From the temperature dependence of the thermal conductivity, we assume radiative cooling dominates for $T<10^{7} \mathrm{~K}$. From the radiative cooling time, $t_{c}=$ $3 \mathrm{KT} / \Lambda_{\gamma}(\mathrm{T}) \mathrm{n}_{\mathrm{e}}$, where $\mathrm{K}$ is the Boltzman constant, $\mathrm{T}$ the plasma temperature before cooling $\left(\sim 2 \times 10^{6} \mathrm{~K}\right), \Lambda_{\gamma}(\mathrm{T})$, the radiative cooling coefficient $\left(10^{-22} \mathrm{erg} \mathrm{cm}^{3} \mathrm{~s}^{-1}\right)$ and $\mathrm{n}_{\mathrm{e}}$ the electron density, we find $\mathrm{n}_{\mathrm{e}} \leq 10^{11} \mathrm{~cm}^{-3}$. The processes involved in heating the plasma at the $\mathrm{X}$-point following coalescence remain uncertain without spectral information and data from other wavelength regimes, especially since the current density might be enhanced also in this region. For example, Dungey (1953) has pointed out that in the vicinity of a magnetic neutral point, a small disturbance can cause the current density to become large in the neighborhood of the neutral point. We have made a first estimate of the energy, $\mathrm{E}$, involved in the reconnection via the coalescence instability (Tajima et al, 1982), in which $E=\left(\mathrm{LB}^{2} \mathrm{a}^{2} / 2\right) \ln (\mathrm{L} / \mathrm{a})$, where $\mathrm{L}$ is the length of the reconnecting region $\left(\sim 7 \times 10^{8} \mathrm{~cm}\right), \quad B$ is the loop magnetic field ( $\sim 100 \mathrm{G})$, and $\mathrm{a}$ is the current channel width $\left(\sim 10^{8} \mathrm{~cm}\right)$, which gives $\mathbf{E}=7 \mathbf{x}$ $10^{28}$ ergs. The numerical values applied here are somewhat uncertain, especially the value of $B$; nevertheless, the value for $E$ is thought to be a reasonable order-of-magnitude estimate, corresponding to that of a very small flare.

\section{References:}

Dungey, J.W.: 1953, Phil. Mag. 44, 725.

Tajima, T., Sakai, J., Nakajima, H., Kosugi, T., Brunel, F. and Kundu, M.R., 1987, Ap. J. 321, 1031.

Tajima, T., Brunel, F. and Sakai, J. 1982, Ap. J., 258, L45. 